Formación docente

revista iberoamericana de educación

\title{
Cultura organizacional y su importancia dentro de la formación en
}

\section{turismo}

Organizational culture and its importance within tourism

Cultura organizacional e sua importância na formação em turismoçç

\section{Josselit Alvari Calva}

Ph.D. Universidad de Pachucha, Pachuca, México. josselitcava@gmail.com, https://orcid.org/0000-0002-8196-0016

Recibido 3 enero 2019 - Aceptado 1 febrero 2020

Formación docente - revista iberoamericana de educación

http://www.revista-iberoamericana.org/index.php/es/index

https://creativecommons.org/licenses/by/4.0/deed.es e-ISSN: $2737-632 \mathrm{X}$

Vol - 3 No. 2, Abril - Junio 2020

Pags 69-81

Resumen En este artículo se encuentra uno de los temas con mayor impacto en el sector turístico, que genera o tiene como finalidad brindar un buen servicio. La cultura organizacional es una parte importante dentro de toda organización o empresa que brinda u ofrece servicios a turistas nacionales y extranjeros, donde se ve integrado los valores como el respeto, honestidad, el trabajo en equipo. El deber que todo empleador tiene con sus empleados para que ellos puedan brindar servicios de calidad. La manera de cómo llegar a enfocarse en sus objetivos que ya se han propuestos, lograr obtener una buena comunicación, apoyo entre el equipo y las áreas de trabajo, existir equidad, responsabilidad y colaboración, que exista un mejor ambiente laboral para que el trabajo sea realizado de la mejor manera y con resultados positivos.

Palabras clave: cultura organizacional, cultura, turismo, servicios, empleador, empleados.

Abstract. In this article we find one of the issues with the greatest impact in the tourism sector, which generates or aims to provide a good service. 
Organizational culture is an important part of any organization or company that offers or offers services to domestic and foreign tourists, where values such as respect, honesty, teamwork are integrated. The duty that every employer has with its employees so that they can provide quality services. The way to get to focus on your objectives that have already been proposed, achieve good communication, support between the team and work areas, there is equity, responsibility and collaboration, there is a better working environment for the work to be performed in the best way and with positive results.

Key words: organizational culture, culture, tourism, services, employer, employees.

Resumo Este artigo contém um dos assuntos com maior impacto no setor do turismo, que gera ou pretende prestar um bom serviço. A cultura organizacional é uma parte importante de qualquer organização ou empresa que presta ou oferece serviços a turistas nacionais e estrangeiros, onde se integram valores como respeito, honestidade e trabalho em equipa. O dever que todo empregador tem com seus empregados para que possam prestar serviços de qualidade. A forma de conseguir focar nos seus objetivos já propostos, conseguir uma boa comunicação, apoio entre a equipa e as áreas de trabalho, existir equidade, responsabilidade e colaboração, que haja um melhor ambiente de trabalho para que o trabalho seja feito da melhor forma e com resultados positivos.

Palavras-chave: cultura organizacional, cultura, turismo, serviços, empregador, empregados. 


\section{INTRODUCCIÓN}

El turismo se ha vuelto un sector de mayor productividad económica dentro de esta sociedad y donde se han hecho cambios para mejorar no solo la atención sino el momento de proceder ante cualquier situación que llegara afectar no solo a la empresa sino quienes colaboran dentro de ella. Para eso debe de existir una buena comunicación entre el empleador y los empleados para lograr buscar soluciones rápidas y factibles. Dentro de este artículo se incorporó la cultura organizacional siendo un conjunto de valores los cuales contribuyan dentro de la empresa.

La expresión cultural organizacional forma parte de las ciencias sociales y, adquirió gran importancia a mitad del siglo XX después de que algunos investigadores en el área de Gestión y Estudios Organizacionales empezaran a defender los beneficios de estudiar la cultura organizacional.

En la cultura organizacional se encuentran las normativas de carácter informal y no escritas, que guían el comportamiento diario de los integrantes de una organización, con el propósito de mejorar el ambiento laboral y el funcionamiento de aquellas empresas que solo se alinean a reglas o normas antiguas y afecta no solo aquella organización sino a todo la parte colaborativa.

La importancia que tiene la cultura organizacional dentro de este artículo es mejorar, capacitar e innovar ideas para el buen manejo de una índole organización que sea equitativa y de resultados positivos. A mejorar también la estabilidad emocional, física y social dentro de lo laboral.

Darle un giro a las actividades organizacionales y desarrollen un mejor trabajo que implica el contacto directo con clientes diariamente, por eso se debe implementar esta cultura dentro de la organización y que genere convencimiento entre los trabajadores para que no sean expuestos a recibirlas 
de manera obligada y eso cause un resultado negativo para el desarrollo y funcionamiento de la empresa.

La cultura organizacional cual hace referencia Schein (Citado por Ritter, 2008) “el conjunto de valores, necesidades, expectativas, creencias, políticas y normas aceptadas y practicadas por los miembros de la organización”.

Se refiere a una gama de valores, conductas y creencias que son compartidas por los mismos miembros de una organización que se obtiene por medio de la práctica diaria, notando las fallas que se puedan visualizar en el momento en que están directamente con el cliente. Su manera de estar en contacto, en cómo se vocaliza, en su forma de actuar, en el trato que brinda cada uno de los empleados. Con eso se puede notar si existe alguna inquietud, pero no debemos dejar o culpar todo a nuestros empleados, quizás nosotros no estamos inculcando de una mejor manera las normas de nuestra empresa.

El lugar donde se labora debe ser agradable no solo para los empleados sino también para el empleador que se sientan cómodos en el área en que ejercen y eso lo explica Davis\& Newstrom (2005) "La cultura se reconoce más fácilmente cuando sus elementos se integran de manera general y son compatibles entre sí, es decir, encajan como piezas de un rompecabezas". Eso es lo que tiene que lograr un empleador que todos sus empleados encajen se sientan cómodos, se entiendan el uno al otro para que aquel trabajo de resultados visibles y positivos.

También hace referencia Davis \& Newstrom (2005) "El conceso en una empresa respecto a su cultura debe generar más cooperación, aceptación de la toma de decisiones y de control, la comunicación y el compromiso con la compañía”. El compromiso, la aceptación que sus empleados tengan al momento de tomar decisiones drásticas para un mejor movimiento laboral, pero no debemos olvidar que dentro de lo laboral debe existir la equidad para que exista un ambiente positivo, confortable así como lo indica (Tziner \& 
Sharoni, 2014, p.36). "Un trabajador espera una retribución equilibrada con los esfuerzos que ellos realizan en las organizaciones, es por esto que requieren de condiciones apropiadas para desempeñarse de la mejor manera”. Se hace referencia a motivaciones que un empleador debería de darle a todos sus empleados por la manera en como ejercen su trabajo, aquella motivación hace que aquel trabajo no sea forzoso sino algo gratificante, brindarles un área cómoda de trabajo, hacerles presentes que gracias a ellos la empresa ha tenido un gran ascenso dentro de lo monetario, pero siempre y cuando ejerzan las normas y culturas que el empleador estableció en su empresa.

La cultura organizacional se construye en el día a día y se refleja en el grado de confianza y lealtad de la gente. Las organizaciones que integran en su estrategia empresarial obtienen una serie de beneficios tales como: un aumento de productividad, una mayor capacidad de contratar personal con un alto potencial, así como una mayor motivación y compromiso del personal con la organización, una disminución del absentismo laboral, una reducción de los estereotipos sexistas, un aumento del nivel de autoestima de las trabajadoras y los trabajadores, un aumento del bienestar general y también una mayor satisfacción laboral (Tsui et al., 1992; Ensher et al., 2001; Foley et al., 2005; Miner-Rubino et al., 2009).

Se refieren al autoestima que un empleado tiene en su área de trabajo, si se obtiene un grupo de trabajadores motivados, leales, que tengan compromiso con la empresa, que se sientan en confianza, pronto se creara un ambiente sano, de energía, donde todos aporten para que aquella organización crezca. "Los procesos de comunicación apropiados facilitan la construcción de relaciones de confianza y transparencia indispensables para el bienestar del empleado" (Hofstede, 1999). Al existir una buena comunicación se crea un vínculo positivo la cual son visibles al momento del trato a un cliente, al estar en un ámbito seguro, en confianza, los empleados demuestran el trato que 
reciben dentro de su organización hacia sus clientes de una manera favorable, la buena atención se debe a como el empleado es tratado en su área laboral.

"El reconocimiento provoca fidelidad, atracción y lealtad en el cliente interno, que es esa pirámide humana que hace parte de la empresa y es la clave del éxito", de acuerdo con Karld Albrecht y Jack Carson autores de los libros La excelencia de los servicios y La revolución de los servicios. Indican el reconocimiento que se le debe dar al empleado que es considerado como la clave del éxito de toda empresa, la manera en cómo actúan ante cualquier situación, su gran habilidad al ejercer aquel trabajo que se lo han dispuesto por su experiencia.

Aquellos que ejercen en horarios distintos, en vacaciones, en festividades, lejos de familias, ellos merecen aquella contribución, reconocimiento y por donde se debe empezar, primeramente por el trato que merecen dentro de su empresa. Si se retroalimenta al trabajador con normas positivas, si se toman el tiempo de escuchar las dudas o inquietudes, se creara un ambiente de confianza. Donde ellos no sientan el trabajo como un ambiente forzoso sino que sientan que es el área que les gusta ejercer, que se sientan en calidad de amigos, crear un bienestar laboral, a no dar tampoco una sobrecarga laboral, antes de aumentar la probabilidad de crecimiento de la empresa, lo que se va a causar es una disminuyo de trabajadores desanimados de su trabajo, así como lo indica Ana Alcayaga "“la creencia de que la sobrecarga laboral es un tema personal de cada trabajador, ha quedado obsoleta. Hoy, este ítem es un factor de riesgo psicosocial, parte de las preocupaciones principales que debe tener una empresa con cada uno de sus colaboradores". La sobrecarga de trabajo a un empleador puede afectar no solo física sino emocionalmente. Debido a tanto trabajo que se le otorga a un solo empleador, este puede verse aturdido, sofocado, no se sentiría bien físicamente, y eso se debe solucionar 
porque toda buena organización debe de entender, es que la base importante que tiene toda empresa son los empleados, y quienes demuestran o brindan la mejor atención a los clientes son específicamente ellos. Y se tiene que mejorar en el trato que se les debe de dar también a quienes aportan dentro de la organización.

No olvidar que toda empresa desea triunfar, pero para lograrlo debe de tener un equipo preparado, dispuesto a colaborar, que sientan su empresa como un espacio de aprendizaje, un ambiente en el cual ellos se sientan cómodos, y los clientes puedan sentirse a su vez gratificantes por el trato que les dan lo empleados. Según (Dávila, 2001; Daft, 2007) reconoce que una empresa exitosa tiene en cuenta en su accionar aspectos generales como creación de buen clima laboral, brindar al personal oportunidades para el crecimiento personal y laboral, mantener transparencia con sus inversionistas, cumplir con las leyes laborales, mantener buenas relaciones con proveedores, contribuir a la conservación del medioambiente, mantener buena relación con la ciudadanía, crear sistemas salariales que permitan la participación de los empleados en las utilidades operacionales e incentivar la promoción cultural de la comunidad".

Dentro de esta organización se evidencia el trabajo excesivo por el que pasan muchos de sus empleados, al ser un establecimiento de hospedaje con una gran demanda turística. Al no ver igualdad, el trabajo se direcciona a un pequeño grupo de trabajadores o hasta solo 1 trabajador.

Cuando un trabajador le asigna la responsabilidad de un trabajo estando solo sin la colaboración de algún otro compañero puede causarle daño físico y disminuir su autoestima. Al no equiparar lo que son las vacaciones, los horarios de jornada, al no existir equidad ni respeto, no podrán conseguir un grupo de empleados leales, colaboradores, con buena energía, que amen su trabajo, sino al contrario se convertirá en un martirio y el ambiente laboral se 
tornara incómodo, no habrá un realce positivo dentro de lo económico, la organización no aspira a la misión y visión.

Lo que se debe realizar para que todos los problemas se solucionen, primero debe existir la comunicación entre el empleador y sus empleados, la equidad, el límite de responsabilidades que debe tener cada uno de los empleados, y lo primordial el respeto. Debe ver más trabajo en equipo ya que todos trabajan por un solo objetivo, todos como departamento deben apoyarse, vender para que puedan lograr tener una rentabilidad para obtener sus utilidades.

Los empleadores deben preocuparse por sus empleados porque ellos realizan el trabajo forzoso, todas las áreas son importantes y los gerentes deben ser el ejemplo, por eso también se debe incorporar una la capacitación para los empleadores no solo para los empleados para que pueda ver una mejor organización dentro de la empresa. El Hotel tiene 5 valores empresariales la cual no todos son puestos en práctica como son:

- Apuntar hacia lo más alto.

- Celebramos las diferencias.

- Hacer lo correcto.

- Trabajo en equipo.

- No existe un yo, sino que somos todos.

\section{MATERIALES Y MÉTODOS}

Behar (2008) explica que el método histórico lógico de investigación se aplica a la disciplina denominada historia, y además, se emplea para asegurar el significado y confiabilidad de hechos pasados en las ciencias en forma general y en cualquier disciplina científica. El método histórico ayuda a establecer las relaciones presentes en los hechos acontecidos en el desarrollo de las ciencias. 
El método establece una forma de evaluar pruebas con el objetivo d determinar hechos históricos, antecedentes que muestren relación con ciencias realizadas en el pasado, para obtener a la final conclusiones que tengan vínculo con evidencias del presente.

A partir del conocimiento general de una realidad realiza la distinción, conocimiento y clasificación de los distintos elementos esenciales que forman parte de ella y de las interrelaciones que sostienen entre sí. Se fundamenta en la premisa de que a partir del todo absoluto se puede conocer y explicar las características de cada una de sus partes y de las relaciones entre ellas.

El método analítico permite aplicar posteriormente el método comparativo, permitiendo establecer las principales relaciones de causalidad que existen entre las variables o factores de la realidad estudiada. Es un método fundamental para toda investigación científica o académica y es necesario para realizar operaciones teóricas como son la conceptualización y la clasificación.

\section{RESULTADOS}

Una encuesta realizada a una población de 30 personas dio como resultado que 23 están totalmente de acuerdo y 7 están de acuerdo.

¿Considera usted que dentro de una empresa debe de existir la cultura organizacional?

Una encuesta realizada a una población de 30 personas dio como resultado que 20 están totalmente de acuerdo, 8 están de acuerdo y 2 ni de acuerdo ni en desacuerdo.

¿Considera usted que todo empleador debe preocuparse por sus empleados? Una encuesta realizada a una población de 30 personas dio como resultado que 23 están totalmente de acuerdo, 6 están de acuerdo y 1 en desacuerdo. 
¿Considera usted que al existir una buena comunicación dentro de la empresa, este se verá reflejado en la atención al cliente?

Una encuesta realizada a una población de 30 personas dio como resultado que 20 están totalmente de acuerdo, 10 están de acuerdo.

¿Considera usted que al incorporar la cultura organizacional dentro del ámbito turístico, ayudara a incrementar el factor económico de la empresa?

Una encuesta realizada a una población de 30 personas dio como resultado que 19 están totalmente de acuerdo, 10 están de acuerdo y 1 ni de acuerdo ni en desacuerdo.

\section{CONCLUSIONES}

Dentro de esta investigación se obtuvo información de distintas personas que dieron su punto de vista hacia el tema, donde la gran parte estuvo totalmente de acuerdo, y que se debería de mejorar la estructura en cuanto cómo se maneja una organización sea pública o privada.

\section{REFERENCIAS}

Arzate, O. (2013). Coaching educativo: una propuesta metodológica para innovar en el aula. Ra Ximhai, 9(4), 177-185.

Barros-Bastidas, C., \& Turpo, O. (2020). La formación en investigación y su incidencia en la producción científica del profesorado de educa- ción de una universidad pública de Ecuador. Publicaciones, 50(2), 167-185. doi:10.30827/publicaciones.v50i2.13952

Barros, C., \& Turpo, O. (2017). La formación en el desarrollo del docente investigador: una revisión sistemática. Revista Espacios, 38(45).

Bou-Pérez, J. (2007). Coaching para docentes. San Vicente - Alicante: Editorial Club Universitario.

Bravo, C. (2013). Pedagogía General (Vol. 1). Quito: Pontífica Universidad Católica del Ecuador.

Castañeda, JG., Camargo, JA y Londoño, MK. (2018). Análisis bibliométrico como herramienta para el seguimiento de instrumentos psicológicos 
validados en Colombia. Revista Ibérica de Sistemas e Tecnologias de Informação, E18, 38-46.

Cubero, H., Visbal, E., \& Olivar, J. (2017). Propuesta para gestionar cambios de evaluación del desempeño. Caso: Universidad Simón Bolívar. Orbis. Revista Científica Ciencias Humanas, 13(38), 19-34.

Daza, J., Castañeda, JG., Tovar, C., Segovia, C y Cortés, JE. (2019). Diseño y análisis psicométrico de una prueba para medir la percepción de clases frente a la formación integral de estudiantes universitarios «PCFI». Espacios, 40 (2), 18-29.

Jiménez, R. (2012). Coaching en el desarrollo profesional docente. Fases formativas y procesos metodológicos de investigación. Revista Electrónica Interuniversitaria de Formación del Profesorado, 15(4), 101-112.

Jiménez, Y., González, M., \& Hernández, J. (2010). Modelo 360 para la evaluación por competencias (enseñanza-aprendizaje). Innovación Educativa, 10(53), 43-53.

Lárez, J. (2008). El coaching educativo como estrategia para potenciar el éxito durante la etapa de desarrollo y culminación del trabajo especial de grado. Sapiens. Revista Universitaria de Investigación, 9(2), 219234.

López Villafranca, P., \& Gómez de Travesedo, R. (2016). Coaching académico para adquirir competencias profesionales. Estudio de caso en la Universidad de Málaga. Opción, 32(10), 95-110.

Malagón, F. (2011). Coaching educativo y académico: un nuevo modo de enseñar y aprender. Educación y Futuro(24), 49-66.

Máñez, C., Navaro, B., \& Bou, J. (2008). Coaching para docentes. (pág. 105). México: CSI.F.

Musicco, G. (2015). Coaching: conciliación de vida laboral-personal (WLB). Revista del Centro de Investigación Universidad La Salle, 11(44), 121-142.

Ortiz, A., Sánchez, J., \& Sánchez, I. (2015). Los modelos pedagógicos desde una dimensión psicológica-espiritual. Revista Científica General José María Córdova, 13(15), 183-194. 
Ospina, Y. (2013). La pedagogía y su incidencia en la formación de sujetos. Hallazgos, 157-170. doi:http://dx.doi.org/10.15332/s17943841.2013.0020.10.

Pimentel, L., \& Rodríguez, A.-M. (2016). El coaching educativo para mejorar la motivación de los docentes. EDUNOVATIC2016 Congreso Virtual Internacional de Educación, Innovación y TIC (pág. 10). Granada: REDINE.

Piñeiro, M., Martinez, M., \& Guillén, E. (2013). Planificación y organización de la docencia enseñar y aprender mediante coaching educativo. Univest, 1-7.

Recalde, H., Cantero, W., \& Jara, J. (2013). Globalización de la educación superior en Paraguay. Revista Gestão Universitária na América Latina - GUAL, 6(4), 37-48.

Rodríguez-Hidalgo, A., Calmaestra, J., \& Maestre, M. (2015). Desarrollo de competencias en el practicum de maestros: ABP y Coaching Multidimensional. Profesorado. Revista de Currículum y Formación de Profesorado, 19(1), 414-434.

Rosa, G., Riberas, G., Navarro-Segura, L., \& Vilar, J. (2015). El Coaching como Herramienta de Trabajo de la Competencia Emocional en la Formación de Estudiantes de Educación Social y Trabajo Social de la Universidad Ramón Llull, España. Formación Universitaria, 8(5), 77-89. doi:http://dx.doi.org/10.4067/S0718-50062015000500009

Ruiz Fernández, M. I., Boada-Grau, J., Merino Tejedor, E., \& Ficapal-Cusí, P. (2014). Una experiencia de coaching en estudiantes universitarios. International Journal of $c ̧$

von Feigenblatt, Otto Federico, ASEAN and Human Security: Challenges and Opportunities (July 29, 2009). Ritsumeikan Center for Asia Pacific Studies Working Paper, No. 09-5, Available at SSRN: https://ssrn.com/abstract=1442476 or http://dx.doi.org/10.213 9/ssrn.1442476

von Feigenblatt, Otto Federico, Japanese Animation as a Global Product: The Lingering Traces of Nijonjinron and the Rise of Globalism and Hybridity (August 2, 2012). Journal of History \& Social Sciences, 2(2), pp. 1-14, July-December 2012, Available at SSRN: $\underline{\text { https://ssrn.com/abstract }=2195562}$ 
von Feigenblatt, Otto Federico, Garcia Marquez' Magical Realism as a Representation of Latin America's Socio-Political Reality: Developmental Simultaneity and Exceptionalism in Latin America as Expressed in Historiographic Metafiction (December 27, 2009). The Expression, Vol. 2, No. 1, pp. 1-6, 2009, Available at SSRN: $\underline{\text { https://ssrn.com/abstract }=1596690}$ 